\title{
Evaluation of Anosmia and Ageusia in COVID-19 Patients: An Observational Study
}

\author{
Monali R Nikalje ${ }^{1}$, Tulsi Subramaniam², Rajkumar Nikalje ${ }^{3}$
}

\begin{abstract}
Aim and objective: Chemosensitive disorders are being reported increasingly in coronavirus disease 2019 (COVID-19) patients. An attempt to document the incidence of this variation in the perception of taste, smell, salivation, and burning sensation in the mouth as well as mouth ulcerations in patients infected with severe acute respiratory syndrome coronavirus 2 (SARS-CoV-2).

Materials and methods: A single-institution short-term observational study was conducted on chemosensitive complaints like ageusia, anosmia, decreased salivation, oral ulcers, and burning sensation in the mouth, in patients diagnosed with COVID-19.

Results: Among 201 patients, $25.9 \%$ of patients reported a loss of smell while $33.3 \%$ of patients reported alteration in taste. $27.9 \%$ of patients reported decreased salivation, while ulcer findings were only in $1.5 \%$ and burning sensation in $1 \%$ of the patients.

Conclusion: Clinicians should suspect infection of SARS-CoV-2 in such patients, to avoid missing diagnosis or misdiagnosis of these patients which could lead to further transmission and spread of the disease.

Clinical significance: Loss of smell and taste seems to be more common in COVID-19 patients as compared to other chemosensitive disorders like ulcers in the mouth or burning sensation or decreased salivation.

Keywords: Ageusia, Anosmia, COVID-19, Smell disorders, Taste disorders.

World Journal of Dentistry (2021): 10.5005/jp-journals-10015-1799
\end{abstract}

\section{INTRODUCTION}

India is reeling under the onslaught of the coronavirus disease 2019 (COVID-19) pandemic along with the rest of the world. India is one of the leading countries with the largest number of confirmed cases in Asia. Early detection and prompt treatment of patients infected with the severe acute respiratory syndrome coronavirus 2 (SARSCoV-2) is one of the key factors for controlling community spread of the infection pandemic.

COVID-19 symptoms which are well documented and reported include fever, cough dyspnea, sputum production, myalgia, arthralgia, headache, diarrhea, nausea, and sore throat. It appears that a subset of COVID-19 patients also develops other symptoms such as ageusia/dysgeusia. ${ }^{1}$ In viral infections, smell and taste dysfunction is a common finding.

Many viruses cause olfactory dysfunction through an inflammatory reaction of the nasal mucosa and the development of rhinorrhea; the common ones being rhinovirus, parainfluenza Epstein-Barr virus, and some coronavirus. ${ }^{2}$ Taste alteration is a common occurrence in many viral infections. It has been proposed that the altered taste in SARS-CoV-2-infected patients, is due to the ability of the virus to bind to the angiotensin-converting enzyme 2 (ACE2) receptor, which is expressed on multiple organ systems, oral cavity, and also the surface of the tongue. ${ }^{1}$ However, rhinitis symptoms and nasal obstruction are not frequent findings in COVID-19 patients. Anosmia and ageusia could probably be due to the damage of olfactory and gustatory receptors by the virus, and the course of recovery of this damage is also not predictable. ${ }^{3}$

A more comprehensive and objective method of evaluating these symptoms is required before one can opine that these are a subset of symptoms accompanying SARS-CoV-2 infection. An alternate explanation for the dysosmia and dysgeusia could be excessive exposure to chemicals and disinfectants that are
1,2Department of Dentistry, Symbiosis Medical College for Women and Symbiosis University Hospital and Research Centre, Symbiosis International (Deemed University), Lavale, Pune, Maharashtra, India

${ }^{3}$ Department of Respiratory Medicine, Symbiosis Medical College for Women and Symbiosis University Hospital and Research Centre, Symbiosis International (Deemed University), Lavale, Pune, Maharashtra, India

Corresponding Author: Monali R Nikalje, Department of Dentistry, Symbiosis Medical College for Women and Symbiosis University Hospital and Research Centre, Symbiosis International (Deemed University), Lavale, Pune, Maharashtra, India, Phone: +91 9960902546, e-mail: monali.nikalje@smcw.siu.edu.in

How to cite this article: Nikalje MR, Subramaniam T, Nikalje R. Evaluation of Anosmia and Ageusia in COVID-19 Patients: An Observational Study. World J Dent 2021;12(1):3-6.

Source of support: Nil

Conflict of interest: None

commonly used by the population due to the viral pandemic, rather than the damage of the olfactory nerve and trigeminal nerve. ${ }^{4}$ An attempt to note the prevalence of these symptoms in COVID-19 patients is presented here.

\section{Materials and Methods}

This study was conducted on SARS-CoV-2 positive patients admitted at Symbiosis University Hospital and Research Center, between June 2020 and September 30, 2020. This research was approved by the Independent Ethics Committee of the Symbiosis International Deemed University as under number SIU/IEC/154. The principles and considerations for the conduct of research involving humans 
were duly followed. The inclusion criteria to be enrolled in the study were; adults (age $>18$ years), patients infected with SARS-CoV-2 virus diagnosed on a real-time PCR test. Exclusion criteria were patients of age $<18$ years, uncooperative patients, critical patients requiring intensive care, psychiatric or neurological disorders, previous trauma, surgery or radiotherapy in the oral and nasal cavities, preexisting test or smell dysfunctions, history of allergic rhinitis or chronic rhinosinusitis, and patients on medications which causes taste alteration. Chemosensory dysfunction, such as, smell and taste disorders are reported as symptoms and are considered as relevant variables for COVID-19 screening. Evaluation of these symptoms can be done as a routine screening procedure with the help of questionnaires without any procedures and interventions. Demographic variables like age, sex, comorbidity, or conditions that could be cause for exclusion were recorded. Patients were screened for initial eligibility depending upon the inclusion and exclusion criteria mentioned above. The screened patients were then verbally questioned about the presence or absence of ageusia and anosmia, decreased salivation, oral ulcers, and burning sensation in the mouth. This information was elicited in the IPD wards of the hospital where the patients were being treated.

\section{Results}

A total of 201 patients, 150 males and 51 females who were positive for coronavirus were screened from June 2020 to September 30, 2020, for complaints of ageusia, anosmia, decreased salivation, oral ulcers, and burning sensation in the mouth. Fifty-two patients reported a loss of smell, while 149 patients did not feel any alterations in smell. Sixty-seven patients reported alteration in taste, while 134 of the others did not. Fifty-six patients reported decreased salivation, while 145 of the others did not. Only 2 patients complained of burning sensation out of 201. Three patients out of 201 complained of oral ulcerations. Summarizing $25.9 \%$ of patients reported loss of smell while $33.3 \%$ of patients reported alteration in taste. $27.9 \%$ of patients reported decreased salivation, while ulcer findings were only in $1.5 \%$ and burning sensation in $1 \%$ of the patients.

The results are summarized in Table 1 and diagrammatically represented in Figure 1.

\section{Discussion}

Anosmia and dysgeusia are symptoms of coronavirus disease in 2019, and anecdotal evidence suggests it could be a new coronavirus causing the disease; with a few reporting altered salivation, burning sensation in the mouth, and ulcers. In this study, $25.9 \%$ of patients reported a loss of smell, while $33.3 \%$ of patients reported alteration in taste. $27.9 \%$ of patients reported decreased salivation, while ulcer findings were only in $1.5 \%$ and burning sensation in $1 \%$ of the patients.

Chary et al. ${ }^{5}$ in their study reported that $70 \%$ of the COVIDpositive patients had olfactory and taste disorders. Agyeman et al. ${ }^{6}$ reported $41 \%$ of patients with olfactory disorders and $38 \%$ of patients with gustatory disturbances after their detailed analysis of 24 studies with data from 8,438 COVID-positive patients. Haehner et al. ${ }^{7}$ reported $64.7 \%$ of patients, who complained of a sudden loss of taste and smell.

Ageusia and anosmia can be the first and only symptomatological manifestation, and patients with anosmia or only age-related anosmia should be compared with people who do not have these symptoms as suggested by Vaira et al. ${ }^{3}$

This will help to improve our understanding of future SARS and CoV-2 infections. Similar findings were reported by Vaira et al. in their comprehensive data analysis too. ${ }^{8}$ It should also be noted that anosmia and ageism can occur due to other reasons, including smoking, drinking, eating, indoor cooking, and smoking outdoors. Other atypical symptoms may indicate infection associated with SARS and CoV-2 infection, such as, fatigue, dizziness, nausea, vomiting, abdominal pain, or diarrhea. The under-representation of symptomatology in a healthy, young population may be due to a lack of awareness of these symptoms and an under-representation of older patients. ${ }^{9,10}$

Further research is needed to show that variants of ACE2 receptor restoration determine clinical manifestations. Data on the prevalence of anosmia in the general population are also needed. To better understand the relationship between SARS and CoV-2 infection and age, we need to study this prospectively, possibly in conjunction with serological tests and with more objective criteria and a larger section of the population. ${ }^{6,11,12}$ The current findings indicate that anosmia and ageism occur as symptoms in patients with COVID-19. ${ }^{9}$

Several studies in Europe have considered anosmia and ageism as symptoms associated with COVID-19 and they were later added. Tanasa et al. ${ }^{12}$ Carrillo-Larco and Altez-Fernandez ${ }^{13}$ analyzed data from $>5,000$ patients who were in taste and smell clinics over four decades and reported a significant association between the symptoms of COVID-19 and the presence of anosmia, senility, and taste disorders.

There are several hypotheses that allow COVID-19 patients to develop anosmia and ageusia, although the exact mechanism for the development of odor loss in patients has not yet been established. There is no evidence that the presence of odor loss may have a direct impact on the ability to smell in patients with COVID19 infection, but there is evidence of an effect of the suspected mechanism in some cases. ${ }^{14}$

COVID-19-associated loss of smell is associated with a very congested nose, and therefore the effect on odor formation can be independent of nasal congestion. ${ }^{13,15}$ The purpose of this report is to summarize emerging COVID-19 symptoms to better inform patients and doctors. The observations made in this study were similar to the ones observed in the European population. ${ }^{15}$ Though there is promising evidence that there are chemosensitive changes like anosmia, ageusia, decreased salivation in SARS-CoV-2 patients, we cannot prematurely conclude that they are definite prognostic or diagnostic indicators of COVID infection.

Table 1: Prevalence of chemosensitive disturbances in COVID-positive patients

\begin{tabular}{llcccc}
\hline & Presence of ulcer & Alteration in smell & Alteration in taste & Decreased salivation & Burning sensation \\
\hline No & 149 & 134 & 145 & 199 \\
Yes & 198 & 52 & 67 & 56 & 02 \\
Total & 03 & 201 & 201 & 201 & 201 \\
\hline
\end{tabular}




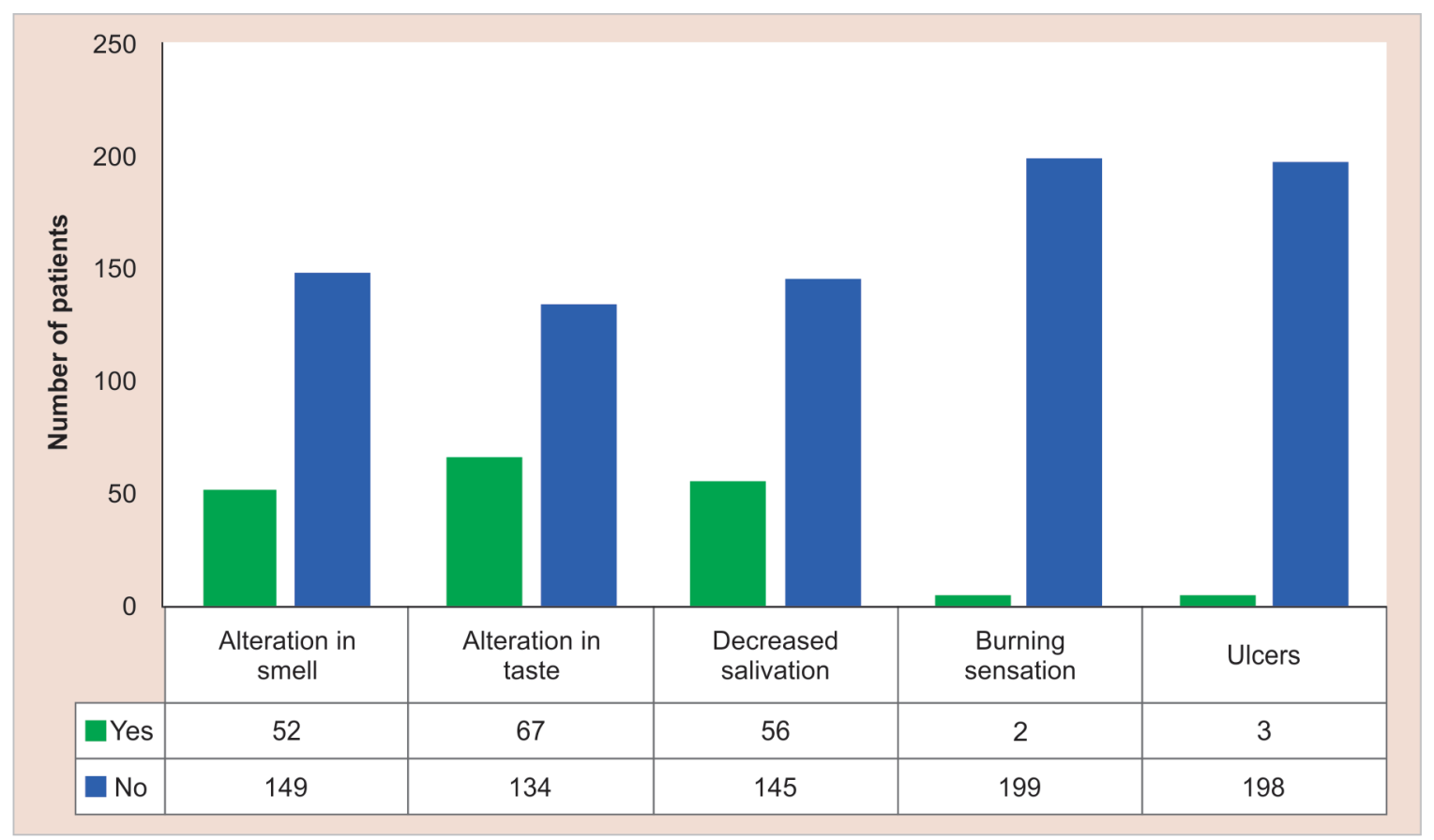

Fig. 1: Prevalence of chemosensory disturbances

\section{Conclusion}

Chemosensitive changes like ageusia and anosmia should raise the suspicion of COVID-19 infection in the mind of the clinician. Missed diagnosis or misdiagnosis can cause further delay in the management and also lead to further transmission of COVID-19 cases. Extensive studies in the early phase are needed in the future to understand a clear picture of the symptomatology of the novel coronavirus which can be included in the screening guidelines.

\section{References}

1. Aziz M, Perisetti A, Lee-Smith WM, et al. Taste changes (dysgeusia) in COVID-19: a systematic review and meta-analysis. Gastroenterology. 2020;159(3):1132-1133. DOI: 10.1053/j.gastro.2020.05.003.

2. Lechien JR, Chiesa-Estomba CM, De Siati DR, et al. Olfactory and gustatory dysfunctions as a clinical presentation of mild-to-moderate forms of the coronavirus disease (COVID-19): a multicenter European study. Eur Arch Otorhinolaryngol 2020;277(8):2251-2261. DOI: 10.1007/s00405-020-05965-1.

3. Vaira LA, Salzano G, Deiana G, et al. Anosmia and ageusia: common findings in COVID-19 patients. Laryngoscope. 2020;130(7):1787. DOI: 10.1002/lary.28692.

4. Keyhan SO, Fallahi HR, Cheshmi B. Dysosmia and dysgeusia due to the 2019 novel coronavirus; a hypothesis that needs further investigation. Maxillofac Plast Reconstr Surg 2020;42(1):9. DOI: 10.1186/s40902-02000254-7.

5. Chary E, Carsuzaa F, Trijolet JP, et al. Prevalence and recovery from olfactory and gustatory dysfunctions in covid-19 infection: a prospective multicenter study. Am J Rhinol Allergy 2020;34(5):686693. DOI: $10.1177 / 1945892420930954$.

6. Agyeman AA, Chin KL, Landersdorfer CB, et al. Smell and taste dysfunction in patients with COVID-19: a systematic review and meta-analysis. Mayo Clin Proc 2020;95(8):1621-1631. DOI: 10.1016/j. mayocp.2020.05.030.

7. Haehner A, Draf J, Dräger S, et al. Predictive value of sudden olfactory loss in the diagnosis of COVID-19. ORL J Otorhinolaryngol Relat Spec 2020;82(4):175-180. DOI: 10.1159/000509143.

8. Vaira LA, Salzano G, Fois AG, et al. Potential pathogenesis of ageusia and anosmia in COVID-19 patients. Int Forum Allergy Rhinol 2020;10(9):1103-1104. DOI: 10.1002/alr.22593.

9. Russell B, Moss C, Rigg A, et al. Anosmia and ageusia are emerging as symptoms in patients with COVID-19: what does the current evidence say. Ecancermedicalscience 2020;14:ed98. DOI: 10.3332/ecancer.2020. ed98.

10. Smith LTAC, Hodges CDRJ, Pratt M, et al. Case report: COVID-19 patient with chief complaint of anosmia and ageusia; a unique perspective on atypical symptomatology and management in the military. Mil Med 2020;185(11-12):e2176-e2179. DOI: 10.1093/milmed/ usaa196.

11. Patel A, Charani E, Ariyanayagam D, et al. New-onset anosmia and ageusia in adult patients diagnosed with SARS-CoV-2 infection. Clin Microbiol Infect. 2020;26(9)):1236-1241. DOI: 10.1016/j. cmi.2020.05.026.

12. Tanasa IA, Manciuc C, Carauleanu A, et al. Anosmia and ageusia associated with coronavirus infection (COVID-19) - what is known. Exp Ther Med 2020;20(3):2344-2347. DOI: 10.3892/etm.2020.8808.

13. Carrillo-Larco RM, Altez-Fernandez C. Anosmia and dysgeusia in COVID-19: a systematic review. Wellcome Open Res 2020;5:94. DOI: 10.12688/wellcomeopenres.15917.1.

14. Zhang $Q$, Shan KS, Abdollahi S, et al. Anosmia and ageusia as the only indicators of coronavirus disease 2019 (COVID-19). Cureus. 2020;12(5):e7918.

15. Lechien JR, Chiesa-Estomba CM, Hans S, et al. Loss of smell and taste in 2013 European patients with mild to moderate COVID-19. Ann Intern Med 2020;173(8):672-675. DOI: 10.7326/M20-2428. 


\section{Mouth Ulcers Assessment Questionnaire}

1. How long have you had mouth ulcers?

2. Where are the mouth ulcers?

3. Have you had mouth ulcers in the past, if yes how frequently and when was the last time you had mouth ulcers?

4. Recent local trauma to the mouth?

5. Dental health?

6. Do you have dentures?

7. Tobacco use?

8. Lip ulceration?

9. Any other skin lesion?

10. Alteration in smell?

11. Alteration in taste?

12. Decrease salivation? 